\title{
Exposure to mesalamine during pregnancy increased preterm deliveries (but not birth defects) and decreased birth weight
}

\author{
Diav-Citrin $O$, Park $Y H$, Veerasuntharam $G$, et al. The safety of mesalamine in human pregnancy: \\ a prospective controlled cohort study. Gastroenterology 1998 fan;114:23-8.
}

\section{Question}

Is mesalamine safe for treating inflammatory bowel disease (IBD) in pregnant women?

Design
Cohort analytic study.

\section{Setting}

A teratogen information service at a children's hospital in Toronto, Canada.

\section{Participants}

165 pregnant women (median age 31 y) who contacted a teratogen information service for information about exposure to mesalamine. 165 women (median age 31 y) who received counselling for exposure during pregnancy to drugs that were not teratogenic were selected as controls from the information service's database. Control participants were matched for history of smoking and alcohol consumption.

\section{Assessment of risk factors}

Telephone and clinic interviews were used to record maternal demographics, clinical histories, details of mesalamine exposure, and concurrent drug exposures.

\section{Main outcome measures}

Outcome data were collected using telephone interviews; physicians were asked, with the mothers' consent, to confirm the medical details. The primary outcome was the incidence of major malformations (structural abnormalities that have serious consequences). Secondary outcomes were pregnancy outcome, maternal weight gain, gestational age at delivery, birth weight, method of delivery, induction of labour, use of forceps, neonatal health problems, and minor malformations.

\begin{abstract}
Main results
Women who took mesalamine only after 13 weeks of gestation were excluded from the analysis of major birth defects. Women who were exposed to mesalamine did not have a greater incidence of major malformations (relative risk [RR] $0.21,95 \%$ CI 0.02 to 1.74 ) or minor malformations (RR 1.65, CI 0.55 to 4.91 ) in their newborns than those exposed to non-teratogens. Compared with women exposed to non-teratogens, those exposed to mesalamine had an increase of $54 \%$ in the preterm delivery rate (CI $18 \%$ to $101 \%$ ) and newborns with a lower birth weight (mean weight $3253 v 3461 \mathrm{~g}$; $\mathrm{p}=0.0005$ ). Women whose IBD was in remission during pregnancy delivered newborns of a higher gestational age than women whose IBD was active (mean age $39.6 v 38.8$ weeks; $p=0.04$ ). Compared with women who were treated with combination therapy, those treated with monotherapy had newborns of a higher gestational age (mean age $39.4 v 38.6$ weeks; $\mathrm{p}=0.02$ ) and birth weight (mean weight $3341 v 3074 \mathrm{~g} ; \mathrm{p}=0.005$ ). No differences existed for the number of live births, induced or spontaneous abortions, ectopic pregnancies, assisted deliveries, neonatal complications, methods of delivery, or rates of foetal distress.
\end{abstract}

\section{Conclusions}

Pregnant women who were exposed to mesalamine did not have more major or minor malformations in their newborns than those exposed to non-teratogens. Compared with non-teratogens, mesalamine led to a lower birth weight and an increase in the preterm delivery rate; the effect for birth weight was greater in women whose inflammatory bowel disease was active during pregnancy and in those who received combination therapy.

Sources of funding: in part, Procter $\mathcal{E}$ Gamble and the Motherisk Research Fund.

For article reprint: Dr G Koren, Division of Clinical Pharmacology and Toxicology, The Hospital for Sick Children, 555 University Avenue, Toronto, ON M5N 1X8, Canada. Fax +14168137562 .

\section{Commentary}

Well-intentioned advice to women with inflammatory bowel disease (IBD) to avoid medications during pregnancy creates more mischief than benefit. Activation of dormant ulcerative colitis or Crohn's disease poses a much greater risk to pregnancy than do aminosalicylates, steroids, or purine antimetabolites. In particular, both the theoretical and the observed obstetrical safety records of sulfasalazine and mesalazine have remained extraordinarily benign throughout the years.

In an effort to make this message even clearer and more authoritative, investigators at The Hospital for Sick Children in Toronto have published a prospective study of 165 pregnant women with IBD on mesalazine therapy, compared with an unspecified but presumably equal number of controls. It is in my view unfortunate that the control group, carefully matched as they were, did not have IBD nor evidently did they have any other important chronic diseases. Thus the cards might have been stacked against mesala- zine in this study - blaming the drug, perhaps, for outcomes actually attributable to the underlying disease.

Nonetheless, the results are reassuring. There were no significant differences between the mesalazine treated patients with IBD and the control women in the numbers of live births, miscarriages, elective terminations, ectopic pregnancies, major malformations, neonatal complications, assisted deliveries, or instances of fetal distress. The mean (SD) birth weight of the babies born to mesalazine treated mothers was statistically significant lower than that of the control babies, but only by

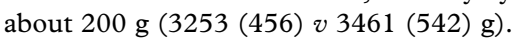
The birth weights were lower in parallel with progressively higher doses of mesalazine, but this apparent dose relation might just have plausibly reflected such confounding variables as concomitant drug treatment (which was independently associated with higher mesalazine doses) or simply more active disease. Indeed, as one might have predicted, the gestational age of the neonates was significantly higher for mothers whose IBD remained in remission throughout the pregnancy than for those who experienced a relapse.

The concluding message of the authors is that "the use of mesalazine in pregnancy is safe". In this respect, mesalazine joins sulfasalazine, steroids, azathioprine, and 6-mercaptopurine as effective agents in IBD that are safe for use during pregnancy. ${ }^{1}$ Pregnant patients and their primary physicians, gastroenterologists, obstetricians, and paediatricians must all be educated to the fact that the greatest risk to pregnancy is active disease, not active treatment.

D SACHAR Division of Gastroenterology, Mount Sinai Medical Center, One Gustave L. Levy Place, New York City, NY 10029, USA

1 Connell WJ. Safety of drug therapy for inflammatory bowel disease in pregnant and nursing matory bowel disease in pregnant and nursing
women. Inflammatory Bowel Disease 1996;2:3347 . 